\title{
Puerarin alleviates liver fibrosis via inhibition of the ERK1/2 signaling pathway in thioacetamide-induced hepatic fibrosis in rats
}

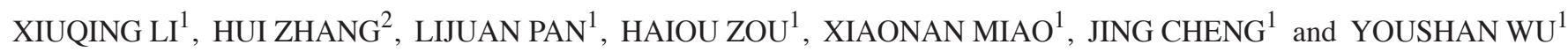 \\ ${ }^{1}$ Department of Gastroenterology and Hepatology, Lianyungang Oriental Hospital, \\ Lianyungang, Jiangsu 222042; ${ }^{2}$ Department of Gastroenterology and Hepatology, \\ The Second Hospital of Lianyungang, Lianyungang, Jiangsu 222023, P.R. China
}

Received October 13, 2017; Accepted March 21, 2019

DOI: $10.3892 /$ etm.2019.7534

\begin{abstract}
Liver fibrosis is a complex pathological process and an early step in the progression of liver cirrhosis, which can eventually develop into hepatocellular carcinoma. Currently, there is no effective treatment for liver fibrosis. Puerarin is a traditional Chinese herb, which is commonly used in the treatment of various diseases. In addition, it is also believed to have a therapeutic effect in liver fibrosis. However, whether puerarin reduces liver fibrosis via the ERK1/2 signaling pathway to inhibit the activation of hepatic stellate cell (HSC) and excessive collagen deposition in liver fibrosis remains unknown. The aim of the current study was to establish a liver fibrosis in vivo model by intraperitoneal injection of thioacetamide (TAA) and investigate the effect of puerarin in the treatment of liver fibrosis. Hematoxylin and eosin and Van Gieson's staining were used to examine histopathological changes associated with liver fibrosis. Liver hydroxyproline content was examined to determine the total amount of collagen in the liver. The relative protein expression levels of transforming growth factor $\beta 1$ (TGF $\beta 1), \alpha$-smooth muscle actin ( $\alpha$-SMA), collagen type I, fibronectin, ERK1/2 and p-ERK1/2 were determined by western blot analysis. In the TAA group, histopathological changes and collagen fiber content in rat liver tissue samples were significantly increased compared with the control group $(\mathrm{P}<0.05)$. In addition, treatment with puerarin significantly decreased histopathological changes and collagen fiber content in rat liver tissue samples $(\mathrm{P}<0.05)$. The relative protein expression levels of TGF $\beta 1, \alpha$-SMA, collagen type I, fibronectin and p-ERK1/2 were significantly upregulated in the TAA group compared with the control group $(\mathrm{P}<0.05)$, whereas puerarin treatment reversed these changes. These findings suggest that treatment with puerarin may reduce HSC activation and
\end{abstract}

Correspondence to: $\mathrm{Dr}$ Youshan Wu, Department of Gastroenterology and Hepatology, Lianyungang Oriental Hospital, 57 Zhonghua West Road, Lianyungang, Jiangsu 222042, P.R. China E-mail:wys2067@126.com

Key words: puerarin, liver fibrosis, hepatic stellate cell, extracellular matrix, ERK1/2 pathway alleviate extracellular matrix protein expression levels by inhibiting the TGF- $\beta /$ ERK1/2 pathway in liver fibrosis.

\section{Introduction}

Various forms of chronic hepatic disease can cause hepatic stellate cell (HSC) activation and induce liver fibrosis, an early step in the progression of liver cirrhosis, which can eventually develop into hepatocellular carcinoma. Notably, these types of hepatic disease are considered a major global health concern (1). Liver fibrosis is a common pathological process, which can eventually lead to liver failure (2). Liver fibrosis is defined as the remodeling and excessive deposition of extracellular matrix (ECM) proteins in the liver (3). The activation of HSC is an important event in the process of liver fibrosis (4). Activated HSCs are commonly regarded as major producers of fibrotic ECM proteins (5), which include collagen type I and fibronectin. Transforming growth factor $\beta 1$ (TGF $\beta 1$ ) is known as the strongest effector in liver fibrosis, which acts as a major pro-fibrotic cytokine, promoting fibroblast recruitment, proliferation and differentiation into ECM-producing myofibroblasts (6). There are several known signaling pathways involved in the progression of liver fibrosis. TGF $\beta 1$ induces liver fibrosis through Smad-dependent and Smad-independent pathways (7), which includes the TGF $\beta 1 /$ ERK1/2 signaling pathway (8). The ERK1/2 pathway has been reported to be important in HSC proliferation and activation via crosstalk with other signaling pathways (9). Notably, a previous study indicated that inhibition of the ERK1/2 signaling pathway enhanced liver fibrosis in rats (10). In addition, it has been demonstrated that the progression of liver fibrosis is reversible (11-14). There are several therapeutic agents, including Orlistat, Metformin and Candesartan, typically used for anti-fibrotic therapy $(15,16)$. However, the major limitation is the lack of effective treatment strategies for liver fibrosis $(17,18)$. Therefore, novel and effective therapeutic targets are required for the treatment of liver fibrosis.

Puerarin is a Chinese herb, which is known to possess several physiological activities, including anti-oxidative and anti-inflammatory activity $(19,20)$. Puerarin has been widely used in the treatment of various diseases, including ischemic stroke (21), renal fibrosis (22), myocardial ischemia and hypertension (23). In addition, puerarin is also considered 
to have a therapeutic effect in liver fibrosis. Previous studies have demonstrated that puerarin can attenuate liver fibrosis by regulating the expression of TGF $\beta 1$ and inhibiting tumor necrosis factor- $\alpha$ (TNF- $\alpha$ )/nuclear factor $\kappa B$ subunit 1 (NF-кB) (24), phosphatidylinositol-4,5-bisphosphate 3-kinase (PI3K)/Akt (25) and TGF $31 /$ Smad (26) signaling pathways. However, whether puerarin reduces liver fibrosis via the ERK1/2 signaling pathway to inhibit the activation of HSCs and excessive collagen deposition in liver fibrosis remains unknown.

Thioacetamide (TAA) is a thiono-sulfur containing compound, which is widely used to induce liver fibrosis in rats to investigate the underlying mechanisms and therapeutic effects of potential anti-fibrotic drugs (27). Therefore, the aim of the current study was to examine the anti-fibrotic efficacy and underlying mechanism of puerarin in TAA-induced liver fibrosis in rats. In addition, changes to the relative protein expression levels of TGF $\beta 1, \alpha$-smooth muscle actin ( $\alpha$-SMA), collagen type I, fibronectin, p-ERK1/2 and ERK1/2 were analyzed.

\section{Materials and methods}

Experimental animals and treatments. A total of 36 male Sprague-Dawley rats (age, 6-8 weeks; weight, 180 200 g) were obtained from Shanxi Medical University Laboratory Animal Center (Taiyuan, China) and used to establish the liver fibrosis in vivo model by intraperitoneal injection of $200 \mathrm{mg} / \mathrm{kg}$ thioacetamide (TAA: Sigma-Aldrich; Merck KGaA). Rats were housed in an environmentally controlled breeding room (12-h dark/light cycle; temperature, $25 \pm 1^{\circ} \mathrm{C}$; humidity, $55 \pm 5 \%$ ) and fed rodent laboratory chow and sterile water. Rats were randomly divided into three groups: Control, TAA and puerarin/TAA group. Rats in the control group received the same dose of PBS once daily $(n=12)$. Rats in the TAA group were injected with $200 \mathrm{mg} / \mathrm{kg}$ TAA three times/week for 8 weeks $(n=12)$. Rats in the puerarin/TAA group were injected with TAA like those in the TAA group, but they also received a daily dose of $0.1 \mathrm{ml} / 10 \mathrm{~g}$ puerarin during week 5 at the same time as TAA administration $(n=12)$. All rats were anesthetized by intraperitoneal injection of chloral hydrate $(400 \mathrm{mg} / \mathrm{kg})$ and sacrificed at week 8 . Liver tissues were harvested and stored at $-80^{\circ} \mathrm{C}$ for further analysis. All animal experiment protocols used in the present study followed internationally accepted principles and were approved by The Institutional Animal Care and Use Committee of Shanxi Medical University (Taiyuan, China).

Immunohistochemistry. Liver tissue was fixed in $10 \%$ neutral buffered formalin for one week at $25-27^{\circ} \mathrm{C}$, dehydrated in a $70-100 \%$ gradient of ethyl alcohol, washed in xylene and embedded in paraffin. Paraffin-embedded liver tissue samples were cut into 4-5 $\mu \mathrm{m}$ thick sections. For general histology, tissue sections were subsequently stained with hematoxylin for $5 \mathrm{~min}$ at room temperature followed by eosin staining for $5 \mathrm{~min}$ at room temperature. Morphological changes were observed via Van Gieson's (VG) staining for $2 \mathrm{~min}$ at room temperature. Pathological changes were observed under a light microscope (magnification, $\mathrm{x} 200$ ). Collagen content was analyzed using the Image-Pro Plus analysis software (version 6.0; Media Cybernetics, Inc., Rockville, MD, USA).
Liver hydroxyproline content analysis. The hydroxyproline content in the liver, as an indirect index of collagen content, was determined using a hydroxyproline testing kit (cat. no. 02140R1; Nanjing Jiancheng Bioengineering Institute, Nanjing, China), according to the manufacturer's protocol. Briefly, liver tissue samples were hydrolyzed, lyophilized and hydroxyproline content was measured at a wavelength of $550 \mathrm{~nm}$ on a spectrophotometer (Molecular Devices, LLC, Sunnyvale, CA, USA).

Western blot analysis. Liver tissue samples were homogenized and total protein was extracted using HEPES extraction buffer (Sangon Biotech, Co., Ltd.). Total protein was quantified using a bicinchoninic acid assay (Santa Cruz Biotechnology, Inc., Dallas, TX, USA) and equal amounts of total protein (40 $\mu \mathrm{g}$ protein/lane) was separated via SDS-PAGE on a $10 \%$ gel. The separated proteins were subsequently transferred onto polyvinyl difluoride membranes and blocked with $3 \%$ skimmed milk for $2 \mathrm{~h}$ at room temperature. The membranes were incubated with primary antibodies against TGF $\beta 1$ (1:1,000; cat. no. ab92486), $\alpha$-SMA (1:1,000; cat. no. ab5694), collagen type I (1:1,000; cat. no. ab34710), fibronectin (1:1,000; cat. no. ab2413), phosphorylated (p-) ERK1/2 (1:1000; cat. no. ab214362), ERK1/2 (1:1,000; cat. no. ab17942) and $\beta$-actin (1:1,000; cat. no. ab8227; all Abcam) overnight at $4^{\circ} \mathrm{C}$. Following primary incubation, membranes were incubated with horseradish peroxidase-conjugated goat anti-rabbit secondary antibody (1:5,000; cat. no. D110066; Sangon Biotech, Co., Ltd.) for $2 \mathrm{~h}$ at room temperature. Protein bands were visualized using the Super ECL detection kit (Bio-Rad Laboratories, Inc., Hercules, CA, USA). Protein expression was quantified using Bio-Rad Quantity One software (version 4.6.2; Bio-Rad Laboratories, Inc.) with $\beta$-actin as the loading control.

Statistical analysis. Data were presented as the mean \pm standard deviation. All statistical analyses were performed using SPSS software (version 16.0; SPSS, Inc., Chicago, IL, USA). One-way analysis of variance followed by Student-Newman-Keuls test was used to analyze differences among treatment groups. $\mathrm{P}<0.05$ was considered to indicate a statistically significant difference.

\section{Results}

Puerarin alleviates inflammation and fibrosis in TAA-induced liver fibrosis in rats. All rats survived the experimental procedure. Liver tissue samples from rats in each experimental group were stained using $\mathrm{H} \& \mathrm{E}$ and VG to examine the histopathological changes associated with liver fibrosis. H\&E staining demonstrated that in the TAA group, there was marked degeneration and necrosis of liver cells, as well as infiltration of inflammatory cells compared with the control group. In addition, the normal structure of the liver was altered, with marked formation of fibrous septum tissue in the TAA group compared with the control group. However, pathological changes in the puerarin/TAA group were less severe compared with the TAA group (Fig. 1A). The presence of collagen type I in the liver tissue was observed by VG staining. In the TAA group, the level of collagen fibers were markedly increased with the formation of fibrous septa surrounding the 

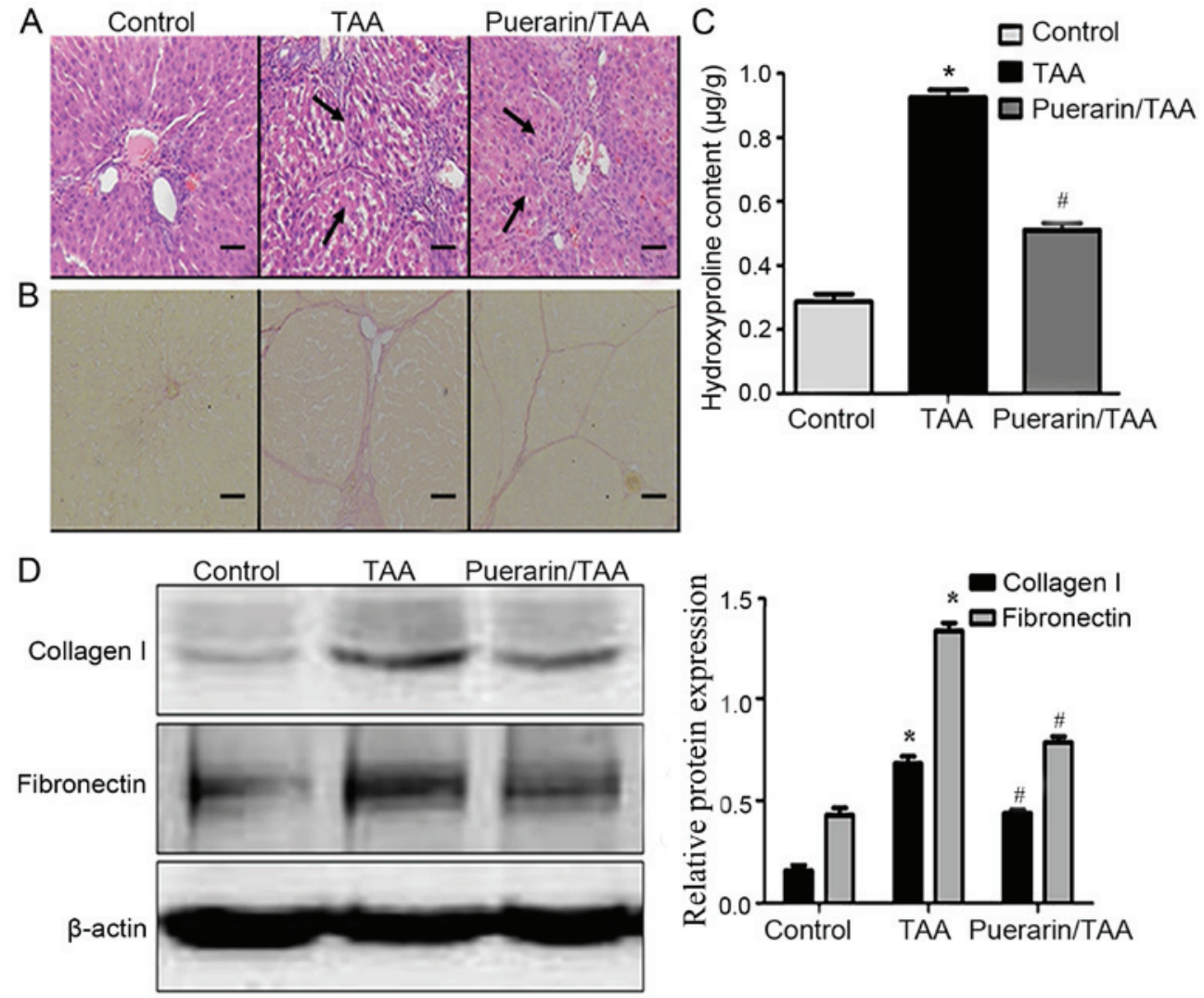

Figure 1. Histopathological changes in the liver fibrosis in vivo model. (A) Hematoxylin and eosin staining was used to examine pathological changes in the rat liver tissue samples of the control, TAA and puerarin/TAA group. The black arrows highlight degeneration and necrosis of liver cells as well as the formation of fibrous septum tissue. (B) Van Gieson's staining was used to examine the presence of collagen type I in the rat liver tissue samples. Scale bar, $50 \mu \mathrm{m}$. (C) Liver hydroxyproline content was examined. (D) Relative protein expression levels of collagen type I and fibronectin were determined by western blot analysis in rat liver tissue samples. $\beta$-actin was used as the loading control $(\mathrm{n}=12)$. ${ }^{*} \mathrm{P}<0.05$ vs. the control group. ${ }^{*} \mathrm{P}<0.05$ vs. the TAA group. TAA, thioacetamide.

hepatic lobules compared with the control group (Fig. 1B). In addition, the collagen deposition in the puerarin/TAA group was less compared with the TAA group (Fig. 1B).

Liver hydroxyproline content was significantly increased in the TAA group compared with the control group $(\mathrm{P}<0.05$; Fig. 1C). In addition, the liver hydroxyproline content significantly decreased in the puerarin/TAA group compared with the TAA group ( $\mathrm{P}<0.05$; Fig. 1C). Similarly, western blot analysis demonstrated that the relative protein expression levels of collagen type I and fibronectin were significantly upregulated in the livers of rats in the TAA group compared with the control group $(\mathrm{P}<0.05$; Fig. 1D). However, the protein expression levels of collagen type I and fibronectin were significantly downregulated in the puerarin/TAA group compared with the TAA group $(\mathrm{P}<0.05$; Fig. 1D). These results suggest that treatment with puerarin may alleviate inflammation and fibrosis in liver fibrosis.

Puerarin alleviates TGFbl expression and HSC activation in TAA-induced liver fibrosis in rats. To further investigate the effects of puerarin on liver fibrosis and HSC activation in vivo following TAA-induced liver fibrosis, the relative expression levels of TGF $\beta 1$ and $\alpha$-SMA were examined. TGF $\beta 1$ is known as the strongest effector for liver fibrosis, while $\alpha$-SMA is a marker of HSC activation $(28,29)$. The relative protein expression levels of TGF $\beta 1$ and $\alpha$-SMA were significantly increased in the TAA group compared with the control group, whereas
TGF $\beta 1$ and $\alpha$-SMA expression levels were significantly decreased in the puerarin/TAA group compared with the TAA group $(\mathrm{P}<0.05$; Fig. $2 \mathrm{~A}$ and $\mathrm{B})$. These results indicated that puerarin may reduce TGF $\beta 1$ production and HSC activation.

Puerarin inhibits the ERK1/2 signaling pathway. The ERK1/2 signaling pathway serves an important role in liver fibrosis $(10,30)$. Therefore, to examine whether puerarin is involved in regulating ERK1/2 in the reduction of HSC activation and ECM deposition, the relative expression levels of ERK1/2 and the activated form, $\mathrm{p}$-ERK1/2, were examined. The relative protein expression level of p-ERK1/2 was markedly increased in the TAA group compared with the control group (Fig. 3A). By contrast, the relative protein expression level of p-ERK1/2 was markedly decreased in the puerarin/TAA group compared with the TAA group (Fig. 3A). The ratio of p-ERK1/2 to ERK1/2 was also significantly decreased in the puerarin/TAA group compared with the TAA group ( $\mathrm{P}<0.05$; Fig. $3 \mathrm{~B})$. There was no marked difference in the protein expression level of ERK1/2 between the experimental groups (Fig. 3A). These results demonstrated that ERK1/2 phosphorylation may be suppressed by puerarin in liver fibrosis.

\section{Discussion}

Liver fibrosis is a major global public health problem (31) and is caused by several forms of chronic liver disease (32). The 

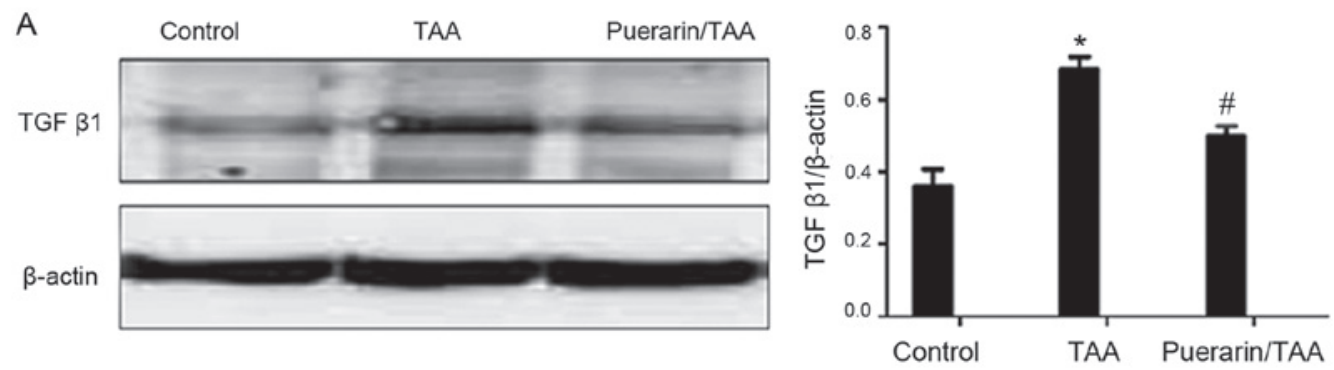

B
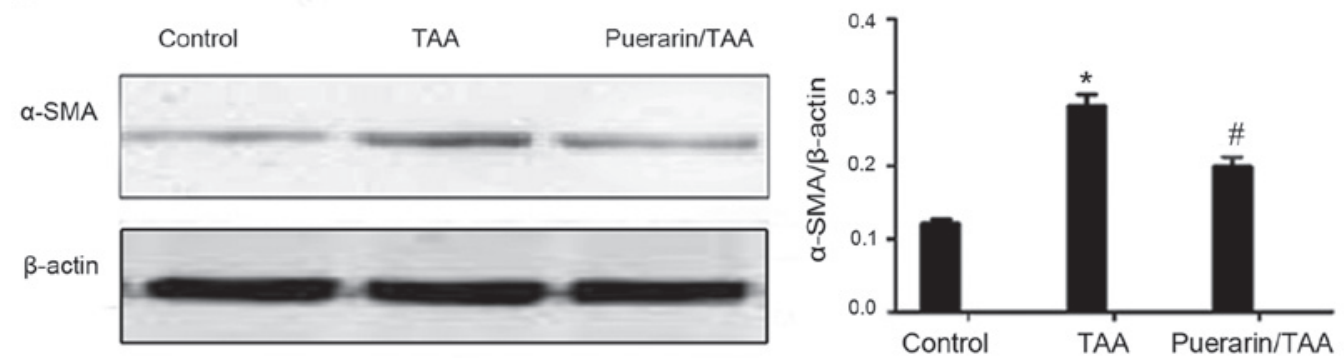

Figure 2. TGF $\beta 1$ and $\alpha$-SMA protein expression. Relative protein expression levels of (A) TGF $\beta 1$ and (B) $\alpha$-SMA were determined by western blot analysis in rat liver tissue samples. $\beta$-actin was used as the loading control $(\mathrm{n}=12) .{ }^{*} \mathrm{P}<0.05$ vs. the control group. ${ }^{~} \mathrm{P}<0.05$ vs. the TAA group. TGF $\beta 1$, transforming growth factor; $\alpha$-SMA, $\alpha$-smooth muscle actin; TAA, thioacetamide.
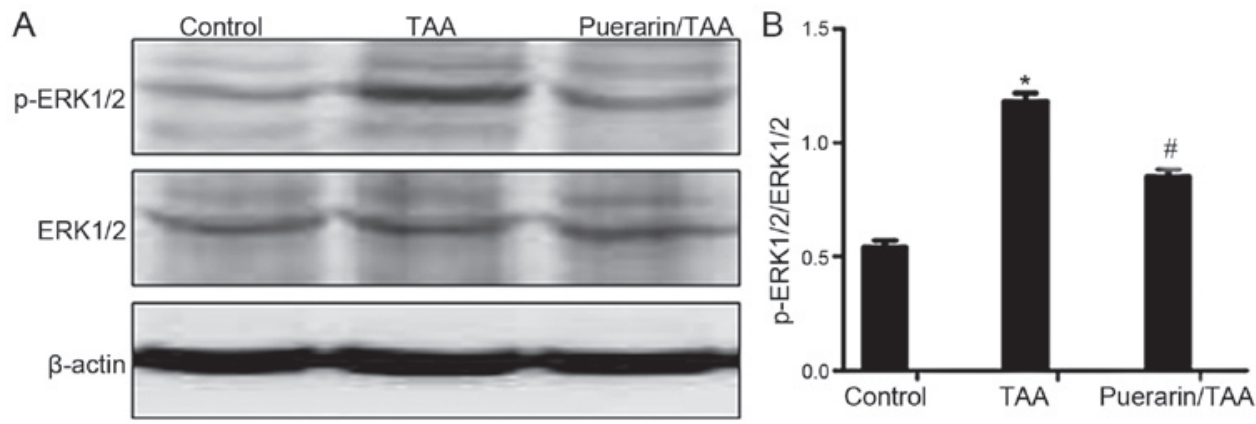

Figure 3. ERK1/2 and p-ERK1/2 expression. (A) Relative protein expression levels of ERK1/2 and p-ERK1/2 were determined by western blot analysis in rat liver tissue samples. (B) Quantification of ERK1/2 and p-ERK1/2 protein expression. $\beta$-actin was used as the loading control ( $\mathrm{n}=12$ ). ${ }^{*} \mathrm{P}<0.05 \mathrm{vs}$. the control group. ${ }^{~} \mathrm{P}<0.05$ vs. the TAA group. TAA, thioacetamide.

pathogenesis of liver fibrosis is complex and is characterized by enhanced ECM production and altered deposition of ECM proteins. Notably, uncontrolled liver fibrosis can develop into cirrhosis (33). Treatment options for liver fibrosis currently available are not highly effective (34). Therefore, more effective treatments for liver fibrosis are required.

TAA can interfere with protein synthesis and enzyme metabolism in the liver, which can lead to chronic liver injury $(35,36)$. Previous studies have demonstrated that TAA could induce hepatic fibrosis in rats $(37,38)$. In the current study, $\mathrm{H} \& \mathrm{E}$ and VG staining revealed a marked increase in the number of necrotic hepatocytes and infiltrating inflammatory cells, with a markedly increased deposition of collagen fibers in the TAA group, as well as liver fibrosis were directly observed. Furthermore, liver hydroxyproline content in the TAA group was significantly increased compared with the control group. These results suggest that the liver fibrosis in vivo model was successfully established in rats.

Chinese herbs, including puerarin, can induce favorable effects in liver fibrosis, whereby ECM components can change from normal basement matrix, which includes collagen type IV, to a fibrotic basement matrix, which includes collagen type I (39). In previous studies, puerarin significantly reduced levels of hydroxyproline and collagen type I in liver fibrosis $(25,26)$. In the current study, hepatic inflammation and the protein expression levels of collagen type I and fibronectin were significantly alleviated following treatment with puerarin, which was consistent with previous reports $(40,41)$. The results from the current study suggest that puerarin may have an anti-fibrotic effect in liver fibrosis. However, the underlying mechanism of puerarin in liver fibrosis remains unknown.

HSC is widely regarded as the key fibrogenic cell that coordinates hepatic ECM formation (5). Activation of HSCs serve an important role in the process of liver fibrosis (4). TGF $\beta 1$ is a potent activator of HSC (42) and a potent inducer of collagen type I production (43). $\alpha$-SMA is a widely accepted marker of activated HSC (44). Previous studies demonstrated that treatment with puerarin reduced the production of TGF- $\beta 1$ and $\alpha$-SMA $(24,45)$. In the current study, protein expression levels of TGF $\beta 1$ and $\alpha$-SMA were significantly 
decreased following treatment with puerarin, which is similar to previous reports $(26,40,45)$. Taken together, these results suggest that puerarin may reduce TGF $\beta 1$ production and $\mathrm{HSC}$ activation to alleviate ECM protein expression in liver fibrosis.

Previous studies revealed that puerarin can regulate serum enzymes, reduce the production of TGF- $\beta 1$ and inhibit excessive collagen deposition to attenuate liver fibrosis via the inhibition of TNF- $\alpha / N F-\kappa B$ (24), PI3K/Akt (25) and TGF $\beta 1 /$ Smad (46) signaling pathways. However, whether puerarin alleviates liver fibrosis via the ERK1/2 signaling pathway remains unknown. Therefore, the current study hypothesized that puerarin may reduce liver fibrosis via the ERK1/2 signaling pathway. In the current study, the ratio of $\mathrm{p}$-ERK1/2 to ERK1/2 protein expression levels were significantly downregulated in the puerarin/TAA group compared with the TAA group, which suggests that the anti-fibrotic effect of puerarin in liver fibrosis may occur via the ERK1/2 signaling pathway. These results suggest that puerarin may ameliorate liver fibrosis by inhibiting the ERK1/2 pathway to reduce HSC activation and ECM deposition. Liu et al (47) reported that puerarin inhibited ERK phosphorylation in breast cancer cells. Therefore, puerarin may be involved in regulating ERK1/2 phosphorylation, thereby inhibiting ERK1/2 signaling in liver fibrosis. However, whether puerarin binds to other functional domains of ERK1/2 requires further investigation.

In conclusion, the current study demonstrated that puerarin may inhibit the TGF- $\beta /$ ERK1/2 signaling pathway to reduce HSC activation, thereby alleviating ECM protein expression in liver fibrosis. Therefore, puerarin may be a potential therapeutic candidate in the treatment of liver fibrosis. However, further investigations are required to validate the potential therapeutic effects of puerarin in liver fibrosis.

\section{Acknowledgements}

Not applicable.

\section{Funding}

The current study was supported by a grant from the Foundation of Lianyungang Oriental Hospital (grant no. 2016-12).

\section{Availability of data and materials}

The datasets used and/or analyzed during the current study are available from the corresponding author on reasonable request.

\section{Authors' contributions}

YW designed the experiments. HaZ, LP, HuZ, XM and JC performed the experiments. XL analyzed the data and prepared the manuscript. All authors read and approved the final manuscript.

\section{Ethics approval and consent to participate}

The study was approved by the Ethics Committee of Shanxi Medical University (SCXK2009-0001).

\section{Patient consent for publication}

Not applicable.

\section{Competing interests}

The authors declare that they have no competing interests.

\section{References}

1. Higashi T, Friedman SL and Hoshida Y: Hepatic stellate cells as key target in liver fibrosis. Adv Drug Deliv Rev 121: 27-42, 2017.

2. Qian H, Deng X, Huang ZW, Wei J, Ding CH, Feng RX, Zeng X, Chen YX, Ding J, Qiu L, et al: An HNF1 $\alpha$-regulated feedback circuit modulates hepatic fibrogenesis via the crosstalk between hepatocytes and hepatic stellate cells. Cell Res 25: 930-945, 2015.

3. Palmer DH, Hussain SA and Johnson PJ: Gene- and immunotherapy for hepatocellular carcinoma. Expert Opin Biol Ther 5: 507-523, 2005.

4. Tao LL, Zhai YZ, Ding D, Yin WH, Liu XP and Yu GY: The role of $\mathrm{C} / \mathrm{EBP}-\alpha$ expression in human liver and liver fibrosis and its relationship with autophagy. Int J Clin Exp Pathol 8: 13102-13107, 2015

5. Lewindon PJ, Pereira TN, Hoskins AC, Bridle KR, Williamson RM, Shepherd RW and Ramm GA: The role of hepatic stellate cells and transforming growth factor-beta(1) in cystic fibrosis liver disease. Am J Pathol 160: 1705-1715, 2002.

6. Sheppard D: Transforming growth factor beta: A central modulator of pulmonary and airway inflammation and fibrosis. Proc Am Thorac Soc 3: 413-417, 2006.

7. Fabregat I, Moreno-Càceres J, Sánchez A, Dooley S, Dewidar B, Giannelli G, Ten Dijke P and IT-LIVER Consortium: TGF- $\beta$ signalling and liver disease. FEBS J 283: 2219-2232, 2016.

8. Cai FF, Wu R, Song YN, Xiong AZ, Chen XL, Yang MD, Yang L, Hu Y, Sun MY and Su SB: Yinchenhao decoction alleviates liver fibrosis by regulating bile acid metabolism and TGF- $\beta /$ Smad/ERK signalling pathway. Sci Rep 8: 15367, 2018.

9. Lestari N, Louisa M, Soetikno V, Suwana AG, Ramadhan PA, Akmal T and Arozal W: Alpha mangostin inhibits the proliferation and activation of acetaldehyde induced hepatic stellate cells through TGF- $\beta$ and ERK 1/2 pathways. J Toxicol 2018: 5360496, 2018.

10. Guo Y, Zhang Y, Zhang Q, Guo X, Zhang H, Zheng G and Liu L: Insulin-like growth factor binding protein-related protein 1 (IGFBPrP1) contributes to liver inflammation and fibrosis via activation of the ERK1/2 pathway. Hepatol Int 9: 130-141, 2015.

11. Benyon RC and Iredale JP: Is liver fibrosis reversible? Gut 46: 443-446, 2000

12. Issa R, Zhou X, Constandinou CM, Fallowfield J, Millward-Sadler H, Gaca MD, Sands E, Suliman I, Trim N, Knorr A, et al: Spontaneous recovery from micronodular cirrhosis: Evidence for incomplete resolution associated with matrix cross-linking. Gastroenterology 126: 1795-1808, 2004.

13. Dienstag JL, Goldin RD, Heathcote EJ, Hann HW, Woessner M, Stephenson SL, Gardner S, Gray DF and Schiff ER: Histological outcome during long-term lamivudine therapy. Gastroenterology 124: 105-117, 2003.

14. Bataller R and Brenner DA: Hepatic stellate cells as a target for the treatment of liver fibrosis. Semin Liver Dis 21: 437-451,2001.

15. Iredale JP, Pellicoro A and Fallowfield JA: Liver fibrosis: Understanding the dynamics of bidirectional wound repair to inform the design of markers and therapies. Dig Dis 35: 310-313, 2017.

16. Schuppan D: Liver fibrosis: Common mechanisms and antifibrotic therapies. Clin Res Hepatol Gastroenterol 39 (Suppl 1): S51-S59, 2015.

17. Friedman SL: Mechanisms of hepatic fibrogenesis. Gastroenterology 134: 1655-1669, 2008.

18. Hernandez-Gea V and Friedman SL: Pathogenesis of liver fibrosis. Annu Rev Pathol 6: 425-456, 2011.

19. Lee JH, Jeon YD, Lee YM and Kim DK: The suppressive effect of puerarin on atopic dermatitis-like skin lesions through regulation of inflammatory mediators in vitro and in vivo. Biochem Biophys Res Commun 498: 707-714, 2018.

20. Chen X, Yu J and Shi J: Management of diabetes mellitus with puerarin, a natural isoflavone from pueraria lobata. Am J Chin Med 46: 1771-1789, 2018. 
21. Yuan M, Liu G, Zheng X, Li P, Liu J, Wang S and Cao Y: Effects of puerarin combined with conventional therapy on ischemic stroke. Exp Ther Med 14: 2943-2946, 2017.

22. Zhou X, Bai C, Sun X, Gong X, Yang Y, Chen C, Shan G and Yao Q: Puerarin attenuates renal fibrosis by reducing oxidative stress induced-epithelial cell apoptosis via MAPK signal pathways in vivo and in vitro. Ren Fail 39: 423-431, 2017.

23. Guo BQ, Xu JB, Xiao M, Ding M and Duan LJ: Puerarin reduces ischemia/reperfusion-induced myocardial injury in diabetic rats via upregulation of vascular endothelial growth factor A/angiotensin-1 and suppression of apoptosis. Mol Med Rep 17: 7421-7427, 2018.

24. Li R, Xu L, Liang T, Li Y, Zhang S and Duan X: Puerarin mediates hepatoprotection against CCl4-induced hepatic fibrosis rats through attenuation of inflammation response and amelioration of metabolic function. Food Chem Toxicol 52: 69-75, 2013.

25. Guo C, Xu L, He Q, Liang T, Duan X and Li R: Anti-fibrotic effects of puerarin on CCl4-induced hepatic fibrosis in rats possibly through the regulation of PPAR- $\gamma$ expression and inhibition of PI3K/Akt pathway. Food Chem Toxicol 56: 436-442, 2013.

26. Xu L, Zheng N, He Q, Li R, Zhang K and Liang T: Puerarin, isolated from Pueraria lobata (Willd.), protects against hepatotoxicity via specific inhibition of the TGF- $\beta 1 /$ Smad signaling pathway, thereby leading to anti-fibrotic effect. Phytomedicine 20 : $1172-1179,2013$

27. Al-Attar AM, Alrobai AA and Almalki DA: Effect of Olea oleaster and Juniperus procera leaves extracts on thioacetamide induced hepatic cirrhosis in male albino mice. Saudi J Biol Sci 23: 363-371, 2016

28. Wickert L, Steinkrüger S, Abiaka M, Bolkenius U, Purps O, Schnabel C and Gressner AM: Quantitative monitoring of the mRNA expression pattern of the TGF-beta-isoforms (beta 1 , beta 2 , beta 3 ) during transdifferentiation of hepatic stellate cells using a newly developed real-time SYBR Green PCR. Biochem Biophys Res Commun 295: 330-335, 2002.

29. Carpino G, Morini S, Ginanni Corradini S, Franchitto A Merli M, Siciliano M, Gentili F, Onetti Muda A, Berloco P, Rossi M, et al: Alpha-SMA expression in hepatic stellate cells and quantitative analysis of hepatic fibrosis in cirrhosis and in recurrent chronic hepatitis after liver transplantation. Dig Liver Dis 37: 349-356, 2005.

30. El-Tanbouly DM, Wadie W and Sayed RH: Modulation of TGF- $\beta /$ Smad and ERK signaling pathways mediates the anti-fibrotic effect of mirtazapine in mice. Toxicol Appl Pharmacol 329: 224-230, 2017.

31. Sánchez-Valle V, Chávez-Tapia NC, Uribe $M$ and Méndez-Sánchez N: Role of oxidative stress and molecular changes in liver fibrosis: A review. Curr Med Chem 19: 4850-4860, 2012.

32. Mormone E, George $\mathrm{J}$ and Nieto N: Molecular pathogenesis of hepatic fibrosis and current therapeutic approaches. Chem Biol Interact 193: 225-231, 2011
33. Elpek GÖ: Cellular and molecular mechanisms in the pathogenesis of liver fibrosis: An update. World J Gastroenterol 20: 7260-7276, 2014

34. Toosi AE: Liver fibrosis: Causes and methods of assessment, a review. Rom J Intern Med 53: 304-314, 2015.

35. Sharma L, Gupta D and Abdullah ST: Thioacetamide potentiates high cholesterol and high fat diet induced steato-hepatitic changes in livers of C57BL/6 J mice: A novel eight weeks model of fibrosing NASH. Toxicol Lett 304: 21-29, 2019.

36. Schyman P, Printz RL, Estes SK, Boyd KL, Shiota M and Wallqvist A: Identification of the toxicity pathways associated with thioacetamide-induced injuries in rat liver and kidney. Front Pharmacol 9: 1272,2018.

37. Amirtharaj GJ, Natarajan SK, Pulimood A, Balasubramanian KA, Venkatraman A and Ramachandran A: Role of oxygen free radicals, nitric oxide and mitochondria in mediating cardiac alterations during liver cirrhosis induced by thioacetamide. Cardiovasc Toxicol 17: 175-184, 2017.

38. GolbarHM,Izawa T, Wijesundera KK, Bondoc A, Tennakoon AH, Kuwamura M and Yamate J: Depletion of hepatic macrophages aggravates liver lesions induced in rats by thioacetamide (TAA). Toxicol Pathol 44: 246-258, 2016.

39. Lakner AM, Moore CC, Gulledge AA and Schrum LW: Daily genetic profiling indicates JAK/STAT signaling promotes early hepatic stellate cell transdifferentiation. World J Gastroenterol 16: 5047-5056, 2010

40. Wang S, Shi XL, Feng M, Wang X, Zhang ZH, Zhao X, Han B, Ma HC, Dai B and Ding YT: Puerarin protects against CCl4-induced liver fibrosis in mice: Possible role of PARP-1 inhibition. Int Immunopharmacol 38: 238-245, 2016.

41. Hou B, Zhao Y, Qiang G, Yang X, Xu C, Chen X, Liu C, Wang X, Zhang $L$ and Du G: Puerarin mitigates diabetic hepatic steatosis and fibrosis by inhibiting TGF- $\beta$ signaling pathway activation in type 2 diabetic rats. Oxid Med Cell Longev 2018: 4545321, 2018.

42. Bataller R and Brenner DA: Liver fibrosis. J Clin Invest 115 209-218, 2005

43. Chen A: Acetaldehyde stimulates the activation of latent transforming growth factor-betal and induces expression of the type II receptor of the cytokine in rat cultured hepatic stellate cells. Biochem J 368: 683-693, 2002.

44. Jin L, Gao H, Wang J, Yang S, Wang J, Liu J, Yang Y, Yan T, Chen T, Zhao Y and He Y: Role and regulation of autophagy and apoptosis by nitric oxide in hepatic stellate cells during acute liver failure. Liver Int 37: 1651-1659, 2017.

45. Wu GL, Chen J, Yu GY, Li JP and Lu WW: Effect of puerarin on levels of TGF-beta1 and alpha-SMA in rats with alcoholic injury liver. Zhongguo Zhong Yao Za Zhi 33: 2245-2249, 2008 (In Chinese)

46. Wu T, Liu T, Xing L and Ji G: Baicalin and puerarin reverse epithelial-mesenchymal transition via the TGF- $\beta 1 / \mathrm{Smad} 3$ pathway in vitro. Exp Ther Med 16: 1968-1974, 2018.

47. Liu X, Zhao W, Wang W, Lin S and Yang L: Puerarin suppresses LPS-induced breast cancer cell migration, invasion and adhesion by blockage NF-kB and Erk pathway. Biomed Pharmacother 92: 429-436, 2017. 